\title{
Dynamical Behavior of a Stochastic Food-Chain System with Beddington-DeAngelis Functional Response
}

\author{
Yanming Ge and Zigen Song \\ College of Information Technology, Shanghai Ocean University, Shanghai 201306, China \\ Correspondence should be addressed to Zigen Song; zigensong@163.com
}

Received 11 March 2014; Revised 13 May 2014; Accepted 18 May 2014; Published 11 June 2014

Academic Editor: Yongli Song

Copyright ( 2014 Y. Ge and Z. Song. This is an open access article distributed under the Creative Commons Attribution License, which permits unrestricted use, distribution, and reproduction in any medium, provided the original work is properly cited.

\begin{abstract}
We investigate a stochastic Food-Chain System $d x(t)=\left[r_{1}(t)-a_{11}(t) x-\left(a_{12}(t) y /\left(1+\beta_{1}(t) x+\gamma_{1}(t) y\right)\right)\right] x d t+\sigma_{1}(t) x d B_{1}(t)$, $d y(t)=\left[r_{2}(t)-a_{21}(t) y+\left(a_{22}(t) x /\left(1+\beta_{1}(t) x+\gamma_{1}(t) y\right)\right)-\left(a_{23}(t) z /\left(1+\beta_{2}(t) y+\gamma_{2}(t) z\right)\right)\right] y d t+\sigma_{2}(t) y d B_{2}(t), d z(t)=\left[-r_{3}(t)+\right.$ $\left.\left(a_{31}(t) y /\left(1+\beta_{2}(t) y+\gamma_{2}(t) z\right)\right)-a_{32}(t) z\right] z d t+\sigma_{3}(t) z d B_{3}(t)$, where $B_{i}(t), i=1,2,3$, is a standard Brownian motion. Firstly, the existence, the uniqueness, and the positivity of the solution are proved. Secondly, the stochastically ultimate boundedness of the system is investigated. Thirdly, the boundedness of moments and upper-growth rate of the solution are obtained. Then the global attractivity of the system is discussed. Finally, the main results are illustrated by several examples.
\end{abstract}

\section{Introduction}

In recent decades, a number of authors have studied the predator-prey type systems [1-8], especially the simple FoodChain Systems [9-14]. These deterministic systems assume that all parameters in the models are deterministic, and some beautiful and valuable conclusions are obtained. Then it is well known that biology systems are often subject to environmental noise [15]. It is therefore useful to reveal how the noise affects these systems. Though there are various types of environmental noise, May [16] have claimed that the birth rates in the system should be stochastic. At the same time, the natural growth of many species vary with time, for example, due to the seasonality.

On the other hand, basing on some natural phenomenon and statistics in laboratory, the predator-dependent functional response should be considered in the predator-prey type systems. As we know, there are three classical predatordependent functional responses: Hassell and Varley [17], Beddington et al. [18, 19], and Crowley and Martin [20]. Recently, Skalski and Gilliam [21] pointed out that these functional responses can provide better descriptions, and the Beddington-DeAngelis (B-D) type functional response performed even better in some cases.
Based on this background, in this paper we will discuss the following stochastic system:

$$
\begin{aligned}
d x(t)= & {\left[r_{1}(t)-a_{11}(t) x-\frac{a_{12}(t) y}{1+\beta_{1}(t) x+\gamma_{1}(t) y}\right] x d t } \\
& +\sigma_{1}(t) x d B_{1}(t), \\
d y(t)= & {\left[r_{2}(t)-a_{21}(t) y+\frac{a_{22}(t) x}{1+\beta_{1}(t) x+\gamma_{1}(t) y}\right.} \\
& \left.-\frac{a_{23}(t) z}{1+\beta_{2}(t) y+\gamma_{2}(t) z}\right] y d t+\sigma_{2}(t) y d B_{2}(t), \\
d z(t)= & {\left[-r_{3}(t)+\frac{a_{31}(t) y}{1+\beta_{2}(t) y+\gamma_{2}(t) z}-a_{32}(t) z\right] z d t } \\
& +\sigma_{3}(t) z d B_{3}(t),
\end{aligned}
$$

where $B_{i}(t), i=1,2,3$, is a Brownian motion defined on a complete probability space $(\Omega, \mathscr{F}, \mathscr{P})$. All parametric functions are continuous and bounded functions on $R_{+} \cdot x(t), y(t)$, and $z(t)$ denote the population density of resource, consumer, and predator at time $t$, respectively. For convenience, 
the model, consisting of (1), together with $x(0)=x_{0}>0$, $y(0)=y_{0}>0, z(0)=z_{0}>0$, will be referred to as model SDE. On the other hand, for easy expression, if $f(t)$ is a continuous bounded function on $R_{+}$, we define $f^{u}=$ $\sup _{t \in R_{+}} f(t)$ and $f^{l}=\inf _{t \in R_{+}} f(t)$. Throughout this paper, we assume that $r_{i}^{l}>0, i=1,2,3$, and the others are similar. The notation $K$ used in this paper expresses the positive constant, for convenience, it denotes different constant in different places, and they only differ in positive constant times.

The rest of the paper is organized as follows. In Section 2, we show that the system has a global positive solution. In Section 3, the stochastically ultimate boundedness of the system is acquired. In Section 4, we obtain the asymptotic moment estimation and the upper-growth rate of the solution. In Section 5, the global attractivity of SDE is investigated. In Section 6, we illustrate our main results through several numerical examples. Last but not least, conclusions are drawn in Section 7.

The key method used in this paper is the analysis of Lyapunov functions. This Lyapunov function analysis for stochastic differential equations was developed by Khasminskii [22] and has been used by many authors [23-28].

\section{Positive and Global Solutions}

As the state of the SDE is the population density of species in the system at time $t$, it should be nonnegative. Moreover, in order for a stochastic differential equation to have a unique global (i.e., no explosion in a finite time) solution for any given initial data, the coefficients of the equation are generally required to satisfy the linear growth condition and local Lipschitz condition [25]. However, the coefficients of each equation in SDE neither obey the linear growth condition nor local Lipschitz continuous. In this section, we show existence and uniqueness of the positive solution.

Lemma 1. For any initial value $x_{0}>0, y_{0}>0, z_{0}>0$, system $(S D E)$ has a unique positive local solution $(x(t), y(t), z(t))$ for $t \in\left[0, \tau_{e}\right)$ almost surely (a.s.), where $\tau_{e}$ is the explosion time.

Proof. To begin with, consider the following equations:

$$
\begin{aligned}
& d u(t) \\
& =\left[b_{1}(t)-a_{11}(t) e^{u(t)}-\frac{a_{12}(t) e^{v(t)}}{1+\beta_{1}(t) e^{u(t)}+\gamma_{1}(t) e^{v(t)}}\right] d t \\
& \quad+\sigma_{1}(t) d B_{1}(t)
\end{aligned}
$$

$d v(t)$

$$
\begin{gathered}
=\left[b_{2}(t)-a_{21}(t) e^{v(t)}+\frac{a_{22}(t) e^{u(t)}}{1+\beta_{1}(t) e^{u(t)}+\gamma_{1}(t) e^{v(t)}}\right. \\
\left.-\frac{a_{23}(t) e^{w(t)}}{1+\beta_{2}(t) e^{v(t)}+\gamma_{2}(t) e^{w(t)}}\right] d t+\sigma_{2}(t) d B_{2}(t),
\end{gathered}
$$

$$
\begin{aligned}
& d w(t) \\
& =\left[-b_{3}(t)+\frac{a_{31}(t) e^{v(t)}}{1+\beta_{2}(t) e^{v(t)}+\gamma_{2}(t) e^{w(t)}}\right. \\
& \left.\quad-a_{32}(t) e^{w(t)}\right] d t+\sigma_{3}(t) d B_{3}(t)
\end{aligned}
$$

on $t \geq 0$ with initial value $u(0)=\ln x_{0}, v(0)=\ln y_{0}$, $w(0)=\ln z_{0}$, where $b_{1}(t)=r_{1}(t)-0.5 \sigma_{1}^{2}(t), b_{2}(t)=r_{2}(t)-$ $0.5 \sigma_{2}^{2}(t), b_{3}(t)=r_{3}(t)+0.5 \sigma_{3}^{2}(t)$. Notice that the last equations' coefficients satisfy the local Lipschitz condition; thus, there is a unique solution $(u(t), v(t), w(t))$ on $t \in\left[0, \tau_{e}\right)$. Therefore, it follows from Itô's formula that $x(t)=e^{u(t)}, y(t)=e^{v(t)}$, $z(t)=e^{w(t)}$ is the unique positive local solution of SDE with initial value $x_{0}>0, y_{0}>0, z_{0}>0$

Theorem 2. Consider system SDE, for any given initial value $\left(x_{0}, y_{0}, z_{0}\right) \in R_{+}^{3}$, and there is a unique solution $(x(t), y(t), z(t))$ on $t \geq 0$ and the solution will remain in $R_{+}^{3}$ with probability 1 , where $R_{+}^{3}=\left\{\left(x_{1}, x_{2}, x_{3}\right) \mid x_{i}>0, i=\right.$ $1,2,3\}$.

Proof. From Lemma 1, to complete the proof we only need to show that $\tau_{e}=\infty$. Hence, let $k_{0}>0$ be sufficiently large for $x_{0}, y_{0}$, and $z_{0}$ lying within the interval $\left[k_{0}^{-1}, k_{0}\right]$. For each integer $k>k_{0}$, define the stopping times:

$$
\begin{gathered}
\tau_{k}=\inf \left\{t \in\left[0, \tau_{e}\right]: x(t) \notin\left(k^{-1}, k\right)\right. \text { or } \\
y(t) \notin\left(k^{-1}, k\right) \text { or } \\
\left.z(t) \notin\left(k^{-1}, k\right)\right\} .
\end{gathered}
$$

Clearly, $\tau_{k}$ is increasing as $k \rightarrow \infty$. Set $\tau_{\infty}=\lim _{k \rightarrow \infty} \tau_{k}$, from which $\tau_{\infty} \leq \tau_{e}$. Now, we only need to prove $\tau_{\infty}=\infty$. If this statement is false, there is a pair of constants $T>0$ and $\epsilon \in(0,1)$ such that $P\left\{\tau_{\infty} \leq T\right\}>\epsilon$. Thus there exists an integer $k_{1} \geq k_{0}$ such that

$$
P\left\{\tau_{k} \leq T\right\} \geq \epsilon, \quad k \geq k_{1} .
$$

Define $V(x, y, z)=x-1-\ln x+y-1-\ln y+z-1-\ln z$. If $(x(t), y(t), z(t)) \in R_{+}^{3}$, we have

$$
\begin{aligned}
& d V(x, y, z) \\
& =(x-1)\left[r_{1}(t)-a_{11}(t) x-\frac{a_{12}(t) y}{1+\beta_{1}(t) x+\gamma_{1}(t) y}\right] d t \\
& +0.5 \sigma_{1}^{2}(t) d t \\
& +(y-1)\left[r_{2}(t)-a_{21}(t) y+\frac{a_{22}(t) x}{1+\beta_{1}(t) x+\gamma_{1}(t) y}\right. \\
& \left.\quad-\frac{a_{23}(t) z}{1+\beta_{2}(t) y+\gamma_{2}(t) z}\right] d t+0.5 \sigma_{2}^{2}(t) d t
\end{aligned}
$$




$$
\begin{aligned}
& +(z-1)\left[-r_{3}(t)+\frac{a_{31}(t) y}{1+\beta_{2}(t) y+\gamma_{2}(t) z}\right. \\
& +(x-1) \sigma_{12}(t) d B_{1}(t)+(y-1) \sigma_{2}(t) d B_{2}(t) \\
& +(z-1) \sigma_{3}(t) d B_{3}(t) \\
& \leq\left\{-a_{11}^{l} x^{2}+\left(r_{1}^{u}+a_{11}^{u}\right) x+\frac{a_{12}^{u}}{r_{1}^{l}}+0.5\left[\sigma_{1}^{u}\right]^{2}\right\} d t \\
& +\left\{-a_{21}^{l} y^{2}+\left(r_{2}^{u}+a_{21}^{u}+\frac{a_{22}^{u}}{\beta_{1}^{l}}\right) y+\frac{a_{23}^{u}}{r_{2}^{l}}+0.5\left[\sigma_{2}^{u}\right]^{2}\right\} d t \\
& +\left\{-a_{32}^{l} z^{2}+\left(\frac{a_{31}^{u}}{\beta_{2}^{l}}+a_{32}^{u}\right) z+r_{3}^{u}+0.5\left[\sigma_{3}^{u}\right]^{2}\right\} d t \\
& +(x-1) \sigma_{1}(t) d B_{1}(t)+(y-1) \sigma_{2}(t) d B_{2}(t) \\
& +(z-1) \sigma_{3}(t) d B_{3}(t) \\
& \leq \\
& + \\
& + \\
& +
\end{aligned}
$$

Integrating both sides of the above inequality from 0 to $\tau_{k} \wedge T$ and then taking the expectations lead to

$$
\begin{gathered}
E V\left(x\left(\tau_{k} \wedge T\right), y\left(\tau_{k} \wedge T\right), z\left(\tau_{k} \wedge T\right)\right) \\
\leq V\left(x_{0}, y_{0}, z_{0}\right)+K T
\end{gathered}
$$

Set $\Omega_{k}=\left\{\tau_{k} \leq T\right\}$; then it follows from inequality (4) that $P\left\{\Omega_{k}\right\} \geq \epsilon$. Clearly, for every $\omega \in \Omega_{k}$, there is $x\left(\tau_{k}, \omega\right)$, $y\left(\tau_{k}, \omega\right)$, or $z\left(\tau_{k}, \omega\right)$ which equals either $k$ or $k^{-1}$; therefore, $V\left(x\left(\tau_{k}, \omega\right), y\left(\tau_{k}, \omega\right), z\left(\tau_{k}, \omega\right)\right)$ is no less than

$$
\min \left\{k-1-\ln k, k^{-1}-1+\ln k\right\} .
$$

Then an application of (6) results in

$$
\begin{aligned}
V\left(x_{0}, y_{0}, z_{0}\right)+K T & \geq E\left[1_{\Omega_{k}} V\left(x\left(\tau_{k}\right), y\left(\tau_{k}\right), z\left(\tau_{k}\right)\right)\right] \\
& \geq \min \left\{k-1-\ln k, k^{-1}-1+\ln k\right\},
\end{aligned}
$$

where $1_{\Omega_{k}}$ is the indicator function of $\Omega_{k}$. Letting $k \rightarrow \infty$ leads to the contradiction

$$
\infty>V\left(x_{0}, y_{0}, z_{0}\right)+K T \geq \infty .
$$

\section{Stochastically Ultimate Boundedness}

In the previous section, we have showed that the solutions of SDE will remain in the positive cone $R_{+}^{3}$ forever. However, this nonexplosion property in a population dynamical system is often not good enough while the property of ultimate boundedness is more desired. Let us discuss it in this section. We begin with giving the definition of stochastically ultimate boundedness.

Definition 3. The SDE is said to be stochastically ultimate bounded if for any $\varepsilon \in(0,1)$, there is a positive constant $H=H(\varepsilon)$ such that for any initial value, the solution of SDE has the property that

$$
\begin{aligned}
\limsup _{t \rightarrow+\infty} P\{|(x(t), y(t), z(t))| \\
\left.=\sqrt{x^{2}(t)+y^{2}(t)+z^{2}(t)}>H\right\}<\varepsilon .
\end{aligned}
$$

Theorem 4. For any $\theta \in(0,1)$ there is a positive constant $\bar{H}=$ $\bar{H}(\theta)$ such that for any initial value, the solution of SDE has the property that

$$
\limsup _{t \rightarrow+\infty} E|(x(t), y(t), z(t))|^{\theta} \leq \bar{H} .
$$

In particular, the SDE is stochastically ultimate bounded.

Proof. Define

$$
V(x, y, z)=x^{\theta}+y^{\theta}+z^{\theta} .
$$

Applying Itôs formula gives

$$
\begin{aligned}
& d V(x, y, z) \\
& =\theta x^{\theta}\left[r_{1}(t)-a_{11}(t) x-\frac{a_{12}(t) y}{1+\beta_{1}(t) x+\gamma_{1}(t) y}\right] d t \\
& +0.5 \theta(\theta-1) x^{\theta} \sigma_{1}^{2}(t) d t \\
& +\theta y^{\theta}\left[r_{2}(t)-a_{21}(t) y+\frac{a_{22}(t) x}{1+\beta_{1}(t) x+\gamma_{1}(t) y}\right. \\
& +0.5 \theta(\theta-1) y^{\theta} \sigma_{2}^{2}(t) d t \\
& +\theta z^{\theta}\left[-r_{3}(t)+\frac{a_{23}(t) z}{1+\beta_{2}(t) y+\gamma_{2}(t) z}-a_{32}(t) z\right] d t \\
& +0.5 \theta(\theta-1) z^{\theta} \sigma_{3}^{2}(t) d t \\
& +\theta x^{\theta} \sigma_{1}(t) d B_{1}(t)+\theta y^{\theta} \sigma_{2}(t) d B_{2}(t)+\theta z^{\theta} \sigma_{3}(t) d B_{3}(t) \\
& +
\end{aligned}
$$




$$
\begin{aligned}
\leq & K d t-V(x, y, z) d t+\theta x^{\theta} \sigma_{1}(t) d B_{1}(t)+\theta y^{\theta} \sigma_{2}(t) d B_{2}(t) \\
& +\theta z^{\theta} \sigma_{3}(t) d B_{3}(t) .
\end{aligned}
$$

Using Itôs formula again we have

$$
\begin{aligned}
& d\left(e^{t} V(x, y, z)\right) \\
& =e^{t}[V(x, y, z) d t+d v(x, y, z)] \\
& \leq e^{t} K d t+e^{t}\left[\theta x^{\theta} \sigma_{1}(t) d B_{1}(t)+\theta y^{\theta} \sigma_{2}(t) d B_{2}(t)\right. \\
& \left.\quad+\theta z^{\theta} \sigma_{3}(t) d B_{3}(t)\right] .
\end{aligned}
$$

Integrating the above inequality and then taking expectations on both sides, we can see that

$$
e^{t} E V(x, y, z) \leq V\left(x_{0}, y_{0}, z_{0}\right)+K\left(e^{t}-1\right) .
$$

This implies immediately that

$$
E V(x, y, z) \leq K .
$$

On the other hand,

$$
\begin{aligned}
|(x(t), y(t), z(t))|^{\theta} & \leq 3^{0.5 \theta} \max \left\{x^{\theta}, y^{\theta}, z^{\theta}\right\} \\
& \leq 3^{0.5 \theta} V(x, y, z),
\end{aligned}
$$

and then we have

$$
\limsup _{t \rightarrow+\infty} E|(x(t), y(t), z(t))|^{\theta} \leq 3^{0.5 \theta} K
$$

which yields the required assertion by setting $\bar{H}=3^{0.5 \theta} \mathrm{K}$. Especially, let $\theta=0.5$; then

$$
\limsup _{t \rightarrow+\infty} E|(x(t), y(t), z(t))|^{0.5} \leq \bar{H} .
$$

Now, for any $\varepsilon \in(0,1)$, let $H=\bar{H}^{2} / \varepsilon^{2}$, by Chebyshev's inequality,

$$
P\{|(x(t), y(t), z(t))|>H\} \leq \frac{E \sqrt{|(x(t), y(t), z(t))|}}{\sqrt{H}} .
$$

Hence,

$$
P\{|(x(t), y(t), z(t))|>H\} \leq \frac{\bar{H}}{\sqrt{H}}=\varepsilon .
$$

In other word, SDE is stochastically ultimate bounded.

\section{Boundedness of Moments and Upper-Growth Rate Estimation}

Theorems 2 and 4 show that the solutions of SDE will remain in a subset of $R_{+}^{3}$. Now let us construct different types of Lyapunov functions to investigate how the solutions behave in $R_{+}^{3}$.
Theorem 5. Consider system SDE for any positive numbers $\rho_{1}, \rho_{2}, \rho_{3} ; \theta_{1}, \theta_{2}, \theta_{3}$, there exists a positive number $K_{2}$ such that, for any initial value $\left(x_{0}, y_{0}, z_{0}\right) \in R_{+}^{3}$, the solution of $S D E$ obeys

$$
\limsup _{t \rightarrow \infty} \frac{E\left[\int_{0}^{t}\left(\rho_{1} x^{\theta_{1}}(s)+\rho_{2} y^{\theta_{2}}(s)+\rho_{3} z^{\theta_{3}}(s)\right) d s\right]}{t} \leq K .
$$

Proof. Define

$$
V(x, y, z)=\rho_{1} x^{\theta_{1}}+\rho_{2} y^{\theta_{2}}+\rho_{3} z^{\theta_{3}} .
$$

Applying Itôs formula gives

$$
\begin{aligned}
d V & (x, y, z) \\
= & \rho_{1} \theta_{1} x^{\theta_{1}}\left[r_{1}(t)-a_{11}(t) x-\frac{a_{12}(t) y}{1+\beta_{1}(t) x+\gamma_{1}(t) y}\right] d t \\
& +0.5 \rho_{1} \theta_{1}\left(\theta_{1}-1\right) x^{\theta_{1}} \sigma_{1}^{2}(t) d t \\
& +\rho_{2} \theta_{2} y^{\theta_{2}}\left[r_{2}(t)-a_{21}(t) y+\frac{a_{22}(t) x}{1+\beta_{1}(t) x+\gamma_{1}(t) y}\right. \\
& \left.\quad-\frac{a_{23}(t) z}{1+\beta_{2}(t) y+\gamma_{2}(t) z}\right] d t \\
& +0.5 \rho_{2} \theta_{2}\left(\theta_{2}-1\right) y^{\theta_{2}} \sigma_{2}^{2}(t) d t \\
& +\rho_{3} \theta_{3} z^{\theta_{3}}\left[-r_{3}(t)+\frac{a_{31}(t) y}{1+\beta_{2}(t) y+\gamma_{2}(t) z}-a_{32}(t) z\right] d t \\
& +0.5 \rho_{3} \theta_{3}\left(\theta_{3}-1\right) z^{\theta_{3}} \sigma_{3}^{2}(t) d t \\
& +\rho_{1} \theta_{1} x^{\theta_{1}} \sigma_{1}(t) d B_{1}(t)+\rho_{2} \theta_{2} y^{\theta_{2}} \sigma_{2}(t) d B_{2}(t) \\
& +\rho_{3} \theta_{3} z^{\theta_{3}} \sigma_{3}(t) d B_{3}(t) \\
\leq & \rho_{1} \theta_{1} x^{\theta_{1}}\left[\theta_{1}^{-1}+r_{1}^{u}-a_{11}^{l} x+0.5 \theta_{1}\left(\sigma_{1}^{u}\right)^{2}\right] d t \\
& +\rho_{2} \theta_{2} y^{\theta_{2}}\left[\theta_{2}^{-1}+r_{2}^{u}-a_{21}^{l} y+\frac{a_{22}^{u}}{\beta_{1}^{l}}+0.5 \theta_{2}\left(\sigma_{2}^{u}\right)^{2}\right] d t \\
& +\rho_{3} \theta_{3} z^{\theta_{3}}\left[\theta_{2}^{-1}+\frac{\left.a_{31}^{u}-a_{32}^{l} z+0.5 \theta_{3}\left(\sigma_{3}^{u}\right)^{2}\right] d t}{\beta_{2}^{l}}\right. \\
& -V(x, y, z) d t+\rho_{1} \theta_{1} x^{\theta_{1}} \sigma_{1}(t) d B_{1}(t) \\
& +\rho_{2} \theta_{2} y^{\theta_{2}} \sigma_{2}(t) d B_{2}(t)+\rho_{3} \theta_{3} z^{\theta_{3}} \sigma_{3}(t) d B_{3}(t) \\
\leq & K d-V(x, y, z) d t+\rho_{1} \theta_{1} x^{\theta_{1}} \sigma_{1}(t) d B_{1}(t) \\
& +\rho_{2} y^{\theta_{2}} \sigma_{2}(t) d B_{2}(t)+\rho_{3} \theta_{3} z^{\theta_{3}} \sigma_{3}(t) d B_{3}(t) . \\
& \\
&
\end{aligned}
$$

Integrating the above inequality and then taking expectations on both sides, we can see that

$$
E V(x, y, z)+E \int_{0}^{t} V(x, y, z) d s \leq K t+V\left(x_{0}, y_{0}, z_{0}\right) .
$$


Together with $E V(x, y, z) \geq 0$, this inequality shows that

$$
E \int_{0}^{t} V(x, y, z) d s \leq K t+V\left(x_{0}, y_{0}, z_{0}\right) .
$$

From this inequality, we obtain the desired assertion.

This theorem, together with Theorem 2, denotes $0 \leq$ $E\left[(1 / t) \int_{0}^{t} x(s) d s\right] \leq K$, in other word, the average value of $x(t)$ on time and "space" is bounded. It is similar to $y(t)$ and $z(t)$.

Theorem 6. For any positive numbers $\alpha_{1}, \alpha_{2}$, and $\alpha_{3}$, solutions of SDE satisfy

$$
\limsup _{t \rightarrow \infty} \frac{\ln \left[x^{\alpha_{1}} y^{\alpha_{2}} z^{\alpha_{3}}\right]}{\ln t} \leq \alpha_{1}+\alpha_{2}+\alpha_{3} \quad \text { a.s. }
$$

Proof. Define

$$
V_{1}(x, y, z)=\alpha_{1} \ln x+\alpha_{2} \ln y+\alpha_{3} \ln z .
$$

Applying Itôs formula gives

$$
\begin{aligned}
& d V_{1}(x, y, z) \\
& =\alpha_{1}\left[b_{1}(t)-a_{11}(t) x-\frac{a_{12}(t) y}{1+\beta_{1}(t) x+\gamma_{1}(t) y}\right] d t \\
& +\alpha_{2}\left[b_{2}(t)-a_{21}(t) y+\frac{a_{22}(t) x}{1+\beta_{1}(t) x+\gamma_{1}(t) y}\right. \\
& \left.\quad-\frac{a_{23}(t) z}{1+\beta_{2}(t) y+\gamma_{2}(t) z}\right] d t \\
& +\alpha_{3}\left[-b_{3}(t)+\frac{a_{31}(t) y}{1+\beta_{2}(t) y+\gamma_{2}(t) z}-a_{32}(t) z\right] d t \\
& +\alpha_{1} \sigma_{1}(t) d B_{1}(t)+\alpha_{2} \sigma_{2}(t) d B_{2}(t) \\
& +\alpha_{3} \sigma_{3}(t) d B_{3}(t) .
\end{aligned}
$$

Let

$$
V_{2}(x, y, z)=e^{\theta t} V_{1}(x, y, z)
$$

where $\theta>0$, and by the Itôs formula, we have

$$
\begin{aligned}
& d V_{2}(x, y, z) \\
& =\theta e^{\theta t} V_{1}(x, y, z) \\
& \quad+\alpha_{1} e^{\theta t}\left[b_{1}(t)-a_{11}(t) x-\frac{a_{12}(t) y}{1+\beta_{1}(t) x+\gamma_{1}(t) y}\right]
\end{aligned}
$$

$$
\begin{aligned}
& +\alpha_{2} e^{\theta t}\left[b_{2}(t)-a_{21}(t) y+\frac{a_{22}(t) x}{1+\beta_{1}(t) x+\gamma_{1}(t) y}\right. \\
& \left.-\frac{a_{23}(t) z}{1+\beta_{2}(t) y+\gamma_{2}(t) z}\right] d t \\
& +\alpha_{3} e^{\theta t}\left[-b_{3}(t)+\frac{a_{31}(t) y}{1+\beta_{2}(t) y+\gamma_{2}(t) z}-a_{32}(t) z\right] d t \\
& +\alpha_{1} e^{\theta t} \sigma_{1}(t) d B_{1}(t)+\alpha_{2} e^{\theta t} \sigma_{2}(t) d B_{2}(t) \\
& +\alpha_{3} e^{\theta t} \sigma_{3}(t) d B_{3}(t) .
\end{aligned}
$$

Therefore,

$$
\begin{aligned}
& V_{2}(x, y, z) \\
& =V_{2}\left(x_{0}, y_{0}, z_{0}\right)+\theta \int_{0}^{t} e^{\theta s} V_{1}(x, y, z) \\
& \quad+\alpha_{1} \int_{0}^{t} e^{\theta s}\left[b_{1}(s)-a_{11}(s) x-\frac{a_{12}(s) y}{1+\beta_{1}(s) x+\gamma_{1}(s) y}\right] d s \\
& \quad+\alpha_{2} \int_{0}^{t} e^{\theta s}\left[b_{2}(s)-a_{21}(s) y+\frac{a_{22}(s) x}{1+\beta_{1}(s) x+\gamma_{1}(s) y}\right. \\
& \quad+\alpha_{3} \int_{0}^{t} e^{\theta s}\left[-b_{3}(s)+\frac{a_{23}(s) z}{1+\beta_{2}(s) y+\gamma_{2}(s) z}\right] d s \\
& +\alpha_{1} \int_{0}^{t} e^{\theta s} \sigma_{1}(s) d B_{1}(s)+\alpha_{2} \int_{0}^{t} e^{\theta s} \sigma_{2}(s) d B_{2}(s) \\
& +\alpha_{3} \int_{0}^{t} e^{\theta s} \sigma_{3}(s) d B_{3}(s) .
\end{aligned}
$$

Define

$$
M_{i}(t)=\int_{0}^{t} e^{\theta s} \sigma_{i}(s) d B_{i}(s), \quad i=1,2,3 .
$$

Notice that the quadratic variation of $M_{i}(t)$ is

$$
\left\langle M_{i}(t), M_{i}(t)\right\rangle=\int_{0}^{t} e^{2 \theta s} \sigma_{i}^{2}(s) d s .
$$

Let $\epsilon \in(0,1)$ and $\lambda>1$. In view of the exponential martingale inequality, for every $k \geq 1$ and $i=1,2,3$, we have

$$
\begin{gathered}
P\left\{\sup _{0 \leq t \leq k}\left[M_{i}(t)-0.5 \epsilon e^{-\theta k}\left\langle M_{i}(t), M_{i}(t)\right\rangle\right]>\frac{\lambda e^{\theta k} \ln k}{\epsilon}\right\} \\
\quad \leq k^{-\lambda} .
\end{gathered}
$$

An application of Borel-Cantelli lemma yields that, for almost any $\omega \in \Omega$, there is a random integer $k_{0}=k_{0}(\omega)$ such that, for $k \geq k_{0}$,

$$
\sup _{0 \leq t \leq k}\left[M_{i}(t)-0.5 \epsilon e^{-\theta k}\left\langle M_{i}(t), M_{i}(t)\right\rangle\right] \leq \frac{\lambda e^{\theta k} \ln k}{\epsilon} .
$$


So

$$
M_{i}(t) \leq 0.5 \epsilon e^{-\theta k} \int_{0}^{t} e^{2 \theta s} \sigma_{i}^{2}(s) d s+\frac{\lambda e^{\theta k} \ln k}{\epsilon}
$$

for $0 \leq t \leq k, k \geq k_{0}$ a.s. Substituting the above inequality into (32), we have

$$
\begin{aligned}
& V_{2}(x, y, z) \\
& \leq V_{2}\left(x_{0}, y_{0}, z_{0}\right)+\theta \int_{0}^{t} e^{\theta s} V_{1}(x, y, z) \\
& +\alpha_{1} \int_{0}^{t} e^{\theta s}\left[b_{1}(s)-a_{11}(s) x-\frac{a_{12}(s) y}{1+\beta_{1}(s) x+\gamma_{1}(s) y}\right. \\
& \left.+0.5 \sigma_{1}^{2}(s) \epsilon e^{\theta(s-k)}\right] d s \\
& +\alpha_{2} \int_{0}^{t} e^{\theta s}\left[b_{2}(s)-a_{21}(s) y+\frac{a_{22}(s) x}{1+\beta_{1}(s) x+\gamma_{1}(s) y}\right. \\
& \quad-\frac{a_{23}(s) z}{1+\beta_{2}(s) y+\gamma_{2}(s) z} \\
& +V_{2}\left(x_{0}, y_{0}, z_{0}\right)+K\left(e^{\theta t}-1\right)+\left(\alpha_{1}+\alpha_{2}+\alpha_{3}\right) \frac{\lambda e^{\theta k} \ln k}{\epsilon} \\
& +\left(\alpha_{1}+\alpha_{2}+\alpha_{3}\right) \frac{\lambda e^{\theta k} \ln k}{\epsilon} \int_{0}^{t} e^{\theta s}\left[-b_{3}(s)+\frac{a_{31}(s) y}{1+\beta_{2}(s) y+\gamma_{2}(s) z}\right. \\
& \left.-a_{32}(s) z+0.5 \sigma_{3}^{2}(s) \epsilon e^{\theta(s-k)}\right] d s \\
& +\alpha^{\theta(s-k)}
\end{aligned}
$$

In other words, we have shown that

$$
\begin{aligned}
& \ln \left[x^{\alpha_{1}} y^{\alpha_{2}} z^{\alpha_{3}}\right] \\
& \quad \leq e^{-\theta t} V_{2}\left(x_{0}, y_{0}, z_{0}\right)+K+\left(\alpha_{1}+\alpha_{2}+\alpha_{3}\right) \frac{\lambda e^{\theta(k-t)} \ln k}{\epsilon} .
\end{aligned}
$$

Thus, for all $k \geq k_{0}, k-1 \leq t \leq k$, we have

$$
\begin{aligned}
\frac{\ln \left[x^{\alpha_{1}} y^{\alpha_{2}} z^{\alpha_{3}}\right]}{\ln t} \leq & \frac{V_{2}\left(x_{0}, y_{0}, z_{0}\right)}{e^{\theta t} \ln t}+\frac{K}{\ln t} \\
& +\frac{\left(\alpha_{1}+\alpha_{2}+\alpha_{3}\right)\left(\lambda e^{\theta(k-t)} \ln k / \epsilon\right)}{\ln (k-1)} .
\end{aligned}
$$

Then

$$
\limsup _{t \rightarrow \infty} \frac{\ln \left[x^{\alpha_{1}} y^{\alpha_{2}} z^{\alpha_{3}}\right]}{\ln t} \leq\left(\alpha_{1}+\alpha_{2}+\alpha_{3}\right) \frac{\lambda e^{\theta}}{\epsilon} .
$$

Letting $\epsilon \rightarrow 1, \lambda \rightarrow 1$, and $\theta \rightarrow 0$, we can obtain the required assertion.
Based on this theorem, obviously, the upper-growth rate is linear.

\section{Global Attractivity}

In this section, we turn to establishing sufficient criteria for the global attractivity of system SDE. Firstly, we prepare some useful lemmas.

Lemma 7. Let $(x(t), y(t), z(t))$ be a solution to SDE with initial value $\left(x_{0}, y_{0}, z_{0}\right) \in R_{+}^{3}$. Then for all $p>1$, there exist three constants related to $p: L_{i}(p)$ for $i=1,2,3$, such that

$$
\begin{aligned}
& \limsup _{t \rightarrow \infty} E\left[x^{p}(t)\right] \leq L_{1}(p), \\
& \limsup _{t \rightarrow \infty} E\left[y^{p}(t)\right] \leq L_{2}(p), \\
& \limsup _{t \rightarrow \infty} E\left[z^{p}(t)\right] \leq L_{3}(p) .
\end{aligned}
$$

Proof. Define $W(y)=y^{p}$ for $y \in R_{+}$. Making use of Itôs formula we have

$$
\begin{aligned}
& d W(y) \\
& =p y^{p}\left[r_{2}(t)-a_{21}(t) y+\frac{a_{22}(t) x}{1+\beta_{1}(t) x+\gamma_{1}(t) y}\right. \\
& \left.\quad-\frac{a_{23}(t) z}{1+\beta_{2}(t) y+\gamma_{2}(t) z}+0.5(p-1) \sigma_{2}^{2}(t)\right] d t \\
& +p \sigma_{2}(t) y^{p} d B_{2}(t) .
\end{aligned}
$$

Applying Itôs formula again to $e^{t} W(y)$ and then taking expectations on both sides, we can see that

$$
\begin{aligned}
& E\left[e^{t} y^{p}(t)\right] \\
& \leq y_{0}^{p}+p \int_{0}^{t} e^{s} y^{p}\left[p^{-1}+r_{2}^{u}-a_{21}^{L} y+\frac{a_{22}^{u}}{\beta_{1}^{l}}+0.5 p\left(\sigma_{2}^{u}\right)^{2}\right] d s \\
& \leq y_{0}^{p}+p K\left(e^{t}-1\right),
\end{aligned}
$$

which is the desired assertion of $\limsup _{t \rightarrow \infty} E\left[y^{p}(t)\right] \leq$ $L_{2}(p)$, and the other two inequalities can be proved similarly.

Lemma 7 tells us that there is a $T>0$, such that $E\left(x^{p}(t)\right) \leq$ $1.5 L_{1}(p), E\left(y^{p}(t)\right) \leq 1.5 L_{2}(p)$, and $E\left(z^{p}(t)\right) \leq 1.5 L_{3}(p)$ for all $t \geq T$. At the same time, it follows from the continuity of $E\left(x^{p}(t)\right), E\left(y^{p}(t)\right)$, and $E\left(z^{p}(t)\right)$ that there exist $\widetilde{L}_{1}(p)>0$, $\widetilde{L}_{2}(p)>0$, and $\widetilde{L}_{3}(p)>0$ such that $E\left(x^{p}(t)\right) \leq \widetilde{L}_{1}(p)$, $E\left(y^{p}(t)\right) \leq \widetilde{L}_{2}(p)$, and $E\left(z^{p}(t)\right) \leq \widetilde{L}_{3}(p)$ for all $0 \leq$ $t \leq T$. Denote $L(p)=\max \left\{1.5 L_{1}(p), \widetilde{L}_{1}(p)\right\}, H(p)=$ $\max \left\{1.5 L_{2}(p), \widetilde{L}_{2}(p)\right\}$, and $G(p)=\max \left\{1.5 L_{3}(p), \widetilde{L}_{3}(p)\right\}$. Then for all $t \geq 0$,

$$
\begin{gathered}
E\left(x^{p}(t)\right) \leq L(p), \quad E\left(y^{p}(t)\right) \leq H(p), \\
E\left(z^{p}(t)\right) \leq G(p) .
\end{gathered}
$$


Lemma 8 (see [29]). Suppose that an n-dimensional stochastic process $X(t)$ on $t \geq 0$ satisfies the condition

$$
E|X(t)-X(s)|^{\beta_{1}} \leq c|t-s|^{1+\beta_{2}}, \quad 0 \leq s, t<\infty,
$$

for some positive constants $\beta_{1}, \beta_{2}$, and $c$. Then there exists a continuous modification $\widetilde{X}(t)$ of $X(t)$ which has the property that for every $v \in\left(0, \beta_{2} / \beta_{1}\right)$ there is a positive random variable $h(\omega)$ such that

$$
\begin{aligned}
& P\left\{\omega: \sup _{0<|t-s|<h(\omega), 0 \leq s, t<\infty} \frac{|\widetilde{X}(t, \omega)-X(t, \omega)|}{|t-s| v} \leq \frac{2}{1-2^{-v}}\right\} \\
& \quad=1 .
\end{aligned}
$$

In other words, almost every sample path of $\widetilde{X}(t)$ is locally but uniformly Hölder continuous with exponent $v$.

Lemma 9. Let $(x(t), y(t), z(t))$ be a solution of SDE on $t \geq 0$ with initial value $\left(x_{0}, y_{0}, z_{0}\right) \in R_{+}^{3}$; then almost every sample path of $(x(t), y(t), z(t))$ is uniformly continuous on $t \geq 0$.

Proof. The second equation of SDE is equivalent to the following stochastic integral equation:

$$
\begin{gathered}
y(t) \\
=y_{0}+\int_{0}^{t} \sigma_{2}(s) y(s) d B_{2}(s) \\
+\int_{0}^{t} y(s)\left[r_{2}(s)-a_{21}(s) y+\frac{a_{22}(s) x}{1+\beta_{1}(s) x+\gamma_{1}(s) y}\right. \\
\left.-\frac{a_{23}(s) z}{1+\beta_{2}(s) y+\gamma_{2}(s) z}\right] d s .
\end{gathered}
$$

We estimate

$$
\begin{aligned}
& E \mid y(s)\left[r_{2}(s)-a_{21}(s) y+\frac{a_{22}(s) x}{1+\beta_{1}(s) x+\gamma_{1}(s) y}\right. \\
& \left.\quad-\frac{a_{23}(s) z}{1+\beta_{2}(s) y+\gamma_{2}(s) z}\right]\left.\right|^{p} \\
& \leq 0.5 E|y(s)|^{2 p} \\
& +0.5 E \mid r_{2}(s)-a_{21}(s) y+\frac{a_{22}(s) x}{1+\beta_{1}(s) x+\gamma_{1}(s) y} \\
& \leq 0.5\left\{H(2 p)+4^{2 p-1}\left[\left(r_{2}^{u}\right)^{2 p}+\left(a_{21}^{u}\right)^{2 p} H(2 p)\right.\right. \\
& \quad-\left.\frac{a_{23}(s) z}{1+\beta_{2}(s) y+\gamma_{2}(s) z}\right|^{2 p} \\
& \left.\left.\quad+\left(\frac{a_{22}^{u}}{\beta_{1}^{l}}\right)^{2 p}+\left(\frac{a_{23}^{u}}{\gamma_{2}^{l}}\right)^{2 p}\right]\right\} \\
& =: C(p) .
\end{aligned}
$$

On the other hand, as an application of the moment inequality for stochastic integrals [30], we see that for $0 \leq$ $t_{1} \leq t_{2}$ and $p>2$,

$$
\begin{aligned}
& E\left|\int_{t_{1}}^{t_{2}} \sigma_{2}(s) y(s) d B_{2}(s)\right|^{p} \\
& \leq\left[\left(\sigma_{2}^{2}\right)^{u}\right]^{p}[0.5 p(p-1)]^{0.5 p}\left(t_{2}-t_{1}\right)^{0.5(p-2)} \int_{t_{1}}^{t_{2}} E|y(s)|^{p} d s \\
& \leq\left[\left(\sigma_{2}^{u}\right)^{2}\right]^{p}[0.5 p(p-1)]^{0.5 p}\left(t_{2}-t_{1}\right)^{0.5 p} H(p) .
\end{aligned}
$$

Then for $0<t_{1}<t_{2}, t_{2}-t_{1} \leq 1, p^{-1}+q^{-1}=1$, we obtain that

$$
\begin{aligned}
& E\left|y\left(t_{2}\right)-y\left(t_{1}\right)\right|^{p} \\
& =E \mid \int_{t_{1}}^{t_{2}} \sigma_{2}(s) y(s) d B_{2}(s) \\
& +\int_{t_{1}}^{t_{2}} y(s)\left[r_{2}(s)-a_{21}(s) y\right. \\
& +\frac{a_{22}(s) x}{1+\beta_{1}(s) x+\gamma_{1}(s) y} \\
& \left.-\frac{a_{23}(s) z}{1+\beta_{2}(s) y+\gamma_{2}(s) z}\right]\left.d s\right|^{p} \\
& \leq 2^{p-1} E \mid \int_{t_{1}}^{t_{2}} y(s)\left[r_{2}(s)-a_{21}(s) y\right. \\
& +\frac{a_{22}(s) x}{1+\beta_{1}(s) x+\gamma_{1}(s) y} \\
& \left.-\frac{a_{23}(s) z}{1+\beta_{2}(s) y+\gamma_{2}(s) z}\right]\left.d s\right|^{p} \\
& +2^{p-1} E\left|\int_{t_{1}}^{t_{2}} \sigma_{2}(s) y(s) d B_{2}(s)\right|^{p} \\
& \leq 2^{p-1}\left(t_{2}-t_{1}\right)^{p} C(p) \\
& +2^{p-1}\left[\left(\sigma_{2}^{u}\right)^{2}\right]^{p}[0.5 p(p-1)]^{0.5 p}\left(t_{2}-t_{1}\right)^{0.5 p} H(p) \\
& \leq 2^{p-1}\left(t_{2}-t_{1}\right)^{0.5 p}\left[1+[0.5 p(p-1)]^{0.5 p}\right] K .
\end{aligned}
$$

Then it follows from Lemma 8 that almost every sample path of $y(t)$ is locally but uniformly Hölder-continuous with exponent $v$ for every $v \in(0,(p-2) / 2 p)$ and therefore almost every sample path of $y(t)$ is uniformly continuous on $t \geq 0$. Similarly, we can show that almost every sample path of $x(t)$ and $z(t)$ is uniformly continuous on $t \geq 0$.

Lemma 10 (see [31]). Let $f$ be a nonnegative function defined on $R_{+}=[0, \infty)$ such that $f$ is integrable on $R_{+}$and is uniformly continuous on $R_{+}$. Then $\lim _{t \rightarrow \infty} f(t)=0$. 
Now, we give our main result of this section.

Definition 11 (see [32]). Let $A_{1}(t)=\left(x_{1}(t), y_{1}(t), z_{1}(t)\right)$ and $A_{2}(t)=\left(x_{2}(t), y_{2}(t), z_{2}(t)\right)$ be two arbitrary solutions of system SDE with initial values $A_{1}(0), A_{2}(0) \in R_{+}^{3}$. If

$$
\begin{aligned}
\lim _{t \rightarrow \infty}\left|x_{1}(t)-x_{2}(t)\right| & =\lim _{t \rightarrow \infty}\left|y_{1}(t)-y_{2}(t)\right| \\
& =\lim _{t \rightarrow \infty}\left|z_{1}(t)-z_{2}(t)\right|=0, \quad \text { a.s., }
\end{aligned}
$$

then we say that the system is globally attractive.

Theorem 12. If $A:=a_{11}^{l}-a_{22}^{u}-a_{12}^{u} \beta_{1}^{u} / \gamma_{1}^{l}>0, B:=a_{21}^{l}-a_{12}^{u}-$ $a_{31}^{u}-a_{23}^{u} \beta_{2}^{u} / \gamma_{2}^{l}-a_{22}^{u} \gamma_{1}^{u} / \beta_{1}^{l}>0, C:=a_{32}^{l}-a_{23}^{u}-a_{31}^{u} \gamma_{2}^{u} / \beta_{2}^{l}>0$, then system SDE is globally attractive.

Proof. Define

$$
\begin{aligned}
V(t)= & \left|\ln x_{1}(t)-\ln x_{2}(t)\right|+\left|\ln y_{1}(t)-\ln y_{2}(t)\right| \\
& +\left|\ln z_{1}(t)-\ln z_{2}(t)\right|
\end{aligned}
$$

then it is a continuous and positive function on $t \geq 0$. A direct calculation of the right differential of $V(t)$, and then applying Itö's formula, we have

$$
\begin{aligned}
& \frac{d^{+} V(t)}{d t} \\
& =\operatorname{sgn}\left(x_{1}-x_{2}\right) \\
& \times\left\{-a_{11}(t)\left(x_{1}-x_{2}\right)+\frac{a_{12}(t) y_{1}}{1+\beta_{1}(t) x_{1}+\gamma_{1}(t) y_{1}}\right. \\
& \left.-\frac{a_{12}(t) y_{2}}{1+\beta_{1}(t) x_{2}+\gamma_{1}(t) y_{2}}\right\} \\
& +\operatorname{sgn}\left(y_{1}-y_{2}\right) \\
& \times\left\{-a_{21}(t)\left(y_{1}-y_{2}\right)+\frac{a_{22}(t) x_{1}}{1+\beta_{1}(t) x_{1}+\gamma_{1}(t) y_{1}}\right. \\
& -\frac{a_{22}(t) x_{2}}{1+\beta_{1}(t) x_{2}+\gamma_{1}(t) y_{2}}-\frac{a_{23}(t) z_{1}}{1+\beta_{2}(t) y_{1}+\gamma_{2}(t) z_{1}} \\
& \left.+\frac{a_{23}(t) z_{2}}{1+\beta_{2}(t) y_{2}+\gamma_{2}(t) z_{2}}\right\} \\
& +\operatorname{sgn}\left(z_{1}-z_{2}\right) \\
& \times\left\{-a_{32}(t)\left(z_{1}-z_{2}\right)+\frac{a_{31}(t) y_{1}}{1+\beta_{2}(t) y_{1}+\gamma_{2}(t) z_{1}}\right. \\
& \left.-\frac{a_{31}(t) y_{2}}{1+\beta_{2}(t) y_{2}+\gamma_{2}(t) z_{2}}\right\}
\end{aligned}
$$

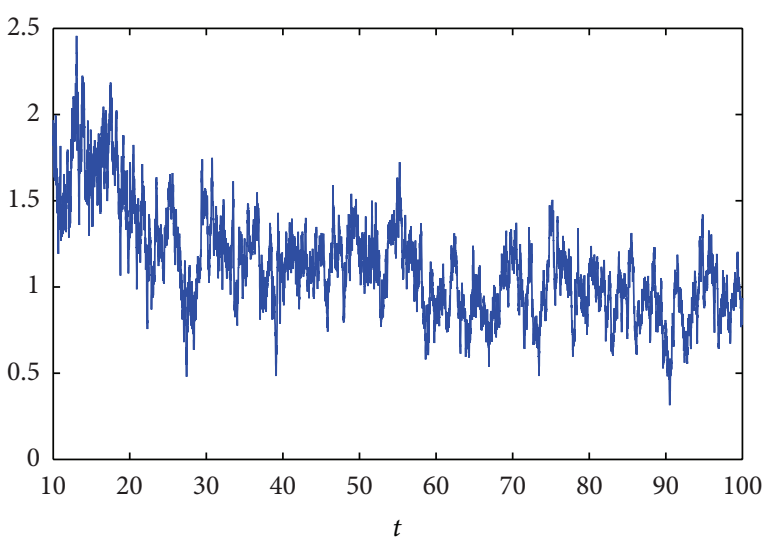

Figure 1: The curve of $\ln [x y z] / \ln t$ for initial values $\left(x_{0}, y_{0}, z_{0}\right)=$ $(0.6,0.5,0.5)$ and $\Delta t=0.01$.

$$
\begin{aligned}
\leq & -a_{11}^{l}\left|x_{1}-x_{2}\right|-a_{21}^{l}\left|y_{1}-y_{2}\right|-a_{32}^{l}\left|z_{1}-z_{2}\right| \\
& +a_{12}^{u} \frac{\beta_{1}^{u}}{\gamma_{1}^{l}}\left|x_{1}-x_{2}\right|+a_{12}^{u}\left|y_{1}-y_{2}\right| \\
& +a_{22}^{u}\left|x_{1}-x_{2}\right|+a_{22}^{u} \frac{\gamma_{1}^{u}}{\beta_{1}^{l}}\left|y_{1}-y_{2}\right| \\
& +a_{23}^{u} \frac{\beta_{2}^{u}}{\gamma_{2}^{l}}\left|y_{1}-y_{2}\right|+a_{23}^{u}\left|z_{1}-z_{2}\right| \\
& +a_{31}^{u} \frac{\gamma_{2}^{u}}{\beta_{2}^{l}}\left|z_{1}-z_{2}\right|+a_{31}^{u}\left|y_{1}-y_{2}\right| \\
= & -A\left|x_{1}-x_{2}\right|-B\left|y_{1}-y_{2}\right|-C\left|z_{1}-z_{2}\right| .
\end{aligned}
$$

Integrating both sides gives

$$
\begin{aligned}
V(t) \leq & V(0) \\
& -\int_{0}^{t}\left[A\left|x_{1}-x_{2}\right|+B\left|y_{1}-y_{2}\right|+C\left|z_{1}-z_{2}\right|\right] d s .
\end{aligned}
$$

Therefore,

$$
\begin{aligned}
V(t) & +\int_{0}^{t}\left[A\left|x_{1}-x_{2}\right|+B\left|y_{1}-y_{2}\right|+C\left|z_{1}-z_{2}\right|\right] d s \\
& \leq V(0)<\infty .
\end{aligned}
$$

Making use of $V(t) \geq 0$ and the known conditions result in that

$$
\begin{aligned}
& \left|x_{1}(t)-x_{2}(t)\right| \in L^{1}[0, \infty), \\
& \left|y_{1}(t)-y_{2}(t)\right| \in L^{1}[0, \infty), \\
& \left|z_{1}(t)-z_{2}(t)\right| \in L^{1}[0, \infty) .
\end{aligned}
$$

Then the required assertion follows from Lemmas 9 and 10 immediately. 

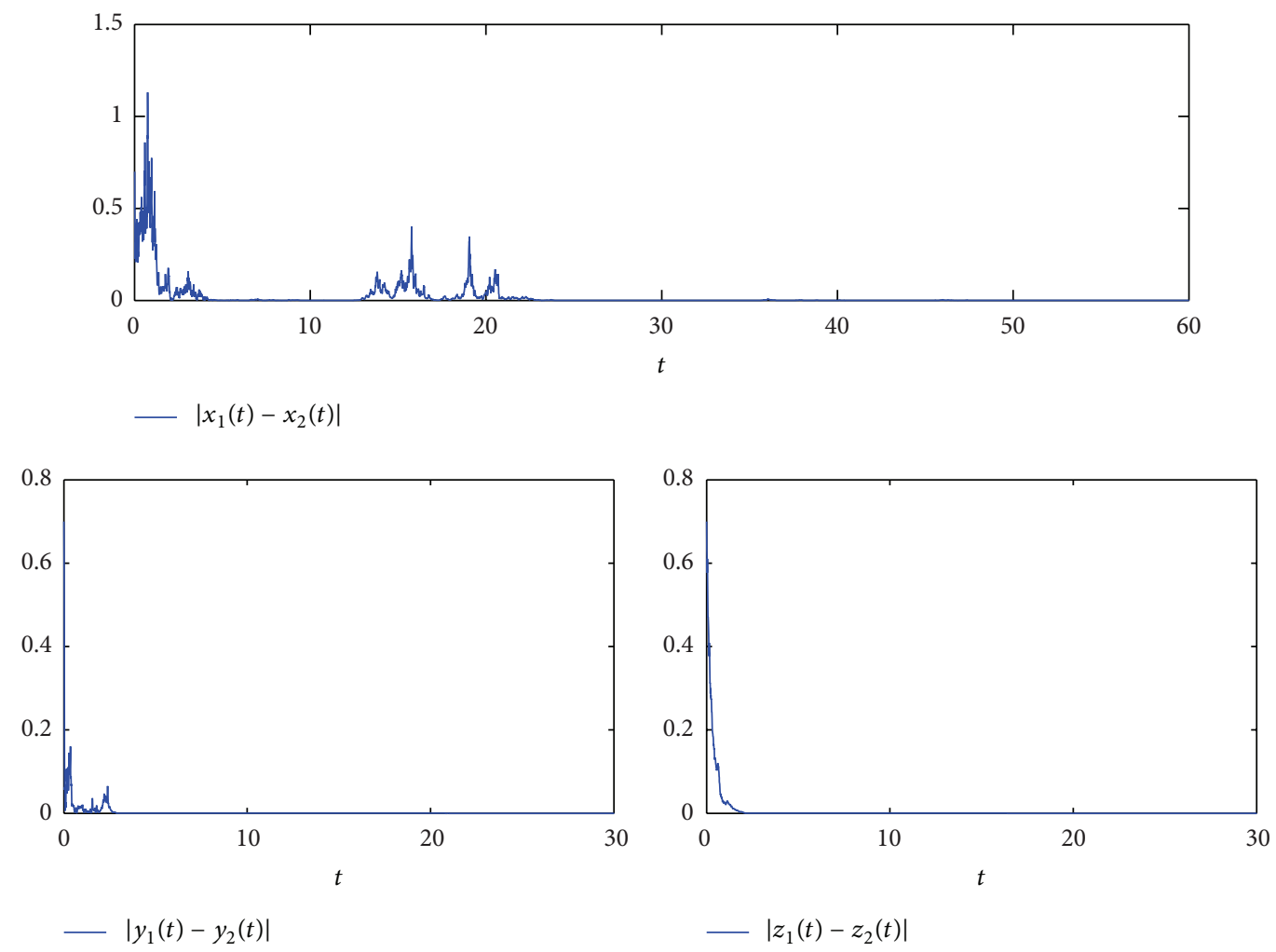

Figure 2: The curves of $\left|x_{1}(t)-x_{2}(t)\right|,\left|y_{1}(t)-y_{2}(t)\right|$ and $\left|z_{1}(t)-z_{2}(t)\right|$ for initial values $\left(x_{1}(0), y_{1}(0), z_{1}(0)\right)=(0.8,0.8,0.8)$ and $\left(x_{2}(0), y_{2}(0), z_{2}(0)\right)=(0.1,0.1,0.1), \Delta t=0.01$.

\section{Numerical Results}

We present numerical experiments in this section to show how the proposed model works in the constructive examples. The results enhance the readers to understand the theoretical conclusions from the practical applications.

Here, we use the Milstein method [33] to construct the discretization equation of SDE. That is,

$$
\begin{aligned}
x_{k+1}= & x_{k} \\
& +x_{k}\left[r_{1}(k \Delta t)-a_{11}(k \Delta t) x_{k}\right. \\
& \left.-\frac{a_{12}(k \Delta t) y_{k}}{1+\beta_{1}(k \Delta t) x_{k}+\gamma_{1}(k \Delta t) y_{k}}\right] \Delta t \\
& +\sigma_{1}(k \Delta t) x_{k} \sqrt{\Delta t} \zeta_{k}+0.5 \sigma_{1}^{2}(k \Delta t) x_{k}^{2}\left(\zeta_{k}^{2}-1\right) \Delta t, \\
& y_{k} \quad+y_{k}\left[r_{2}(k \Delta t)-a_{21}(k \Delta t) y_{k}\right. \\
& +\frac{a_{22}(k \Delta t) x_{k}}{1+\beta_{1}(k \Delta t) x_{k}+\gamma_{1}(k \Delta t) y_{k}} \\
& \left.-\frac{a_{23}(k \Delta t) z_{k}}{1+\beta_{2}(k \Delta t) y_{k}+\gamma_{2}(k \Delta t) z_{k}}\right] \Delta t \\
+ & \sigma_{2}(k \Delta t) y_{k} \sqrt{\Delta t} \eta_{k}+0.5 \sigma_{2}^{2}(k \Delta t) y_{k}^{2}\left(\eta_{k}^{2}-1\right) \Delta t,
\end{aligned}
$$

$$
z_{k+1}=z_{k}
$$$$
\begin{array}{r}
+z_{k}\left[-r_{3}(k \Delta t)+\frac{a_{31}(k \Delta t) y_{k}}{1+\beta_{2}(k \Delta t) y_{k}+\gamma_{2}(k \Delta t) z_{k}}\right. \\
\left.-a_{32}(k \Delta t) z_{k}\right] \Delta t+\sigma_{3}(k \Delta t) z_{k} \sqrt{\Delta t} \xi_{k}
\end{array}
$$$$
+0.5 \sigma_{3}^{2}(k \Delta t) z_{k}^{2}\left(\xi_{k}^{2}-1\right) \Delta t
$$

where $\zeta_{k}, \eta_{k}$, and $\xi_{k}, k=1, \ldots, n$, are the Gaussian random variables. We obtain the following results.

As we pointed out in Theorem 6, $\lim \sup _{t \rightarrow \infty} \ln$ $\left[x^{\alpha_{1}} y^{\alpha_{2}} z^{\alpha_{3}}\right] / \ln t \leq \alpha_{1}+\alpha_{2}+\alpha_{3}$ a.s., and Figure 1 confirms this. Here we choose $r_{1}(t)=9+\sin (t), r_{2}(t)=5+\sin (t)$, $r_{3}(t)=0.1+0.05 \sin (t), a_{11}(t)=a_{21}(t)=1+0.1 \sin (t)$, $a_{12}(t)=a_{22}(t)=0.4+0.2 \sin (t), a_{23}(t)=0.1+0.05 \sin (t)$, $a_{31}=5+0.05 \sin (t), a_{32}(t)=0.5+0.1 \sin (t), \beta_{1}(t)=\gamma_{1}(t)=1$, $\beta_{2}(t)=\gamma_{2}(t)=2, \sigma_{1}(t)=\sigma_{2}(t)=\sigma_{3}(t)=1+0.1 \sin (t)$, and $\alpha_{1}=\alpha_{2}=\alpha_{3}=1$.

Figure 2 confirms that the system is globally attractive (Theorem 12). Here $r_{1}(t)=1.1+\sin (t), r_{2}(t)=0.1+$ $0.05 \sin (t), r_{3}(t)=2+0.1 \sin (t), a_{11}(t)=a_{21}(t)=10.1+$ $0.1 \sin (t), a_{12}(t)=a_{22}(t)=a_{23}(t)=a_{31}(t)=0.8+0.2 \sin (t)$, $a_{32}(t)=4+\sin (t), \beta_{1}(t)=\gamma_{1}(t)=1, \beta_{2}(t)=\gamma_{2}(t)=10$, $\sigma_{1}(t)=\sigma_{2}(t)=2+0.1 \sin (t), \sigma_{3}(t)=0.4+0.1 \sin (t)$. 
The initial values are $\left(x_{1}(0), y_{1}(0), z_{1}(0)\right)=(0.8,0.8,0.8)$ and $\left(x_{2}(0), y_{2}(0), z_{2}(0)\right)=(0.1,0.1,0.1)$. Then $A=8, B=6$, $C=1$, and the assumptions of Theorem 12 are satisfied.

\section{Conclusions}

This paper studies a stochastic nonautonomous food-chain system with Beddington-DeAngelis functional response. This system retains some conventional properties of stochastic systems. That is, it has a unique global positive solution for any initial conditions, the moments of the solutions are bounded, and the solutions are global attractive and stochastically ultimate bounded.

Nevertheless, there are rooms to continue working on this issue. It is interesting to study other parameters; for example, $a_{i j}(t)$ are stochastic. The permanence and extinction of the system and the stability in distribution deserve to be investigated too.

\section{Conflict of Interests}

The authors declare that there is no conflict of interests regarding the publication of this paper.

\section{Acknowledgment}

This research is supported by the National Natural Science Foundation of China under Grant no. 11302126.

\section{References}

[1] C. Azar, J. Holmberg, and K. Lindgren, "Stability analysis of harvesting in a predator-prey model," Journal of Theoretical Biology, vol. 174, no. 1, pp. 13-19, 1995.

[2] M. R. Myerscough, M. J. Darwen, and W. L. Hogarth, "Stability, persistence and structural stability in a classical predator-prey model," Ecological Modelling, vol. 89, no. 1-3, pp. 31-42, 1996.

[3] J. A. Axelsen, N. Holst, T. Hamers, and P. H. Krogh, "Simulations of the predator prey interactions in a two species ecotoxicological test system," Ecological Modelling, vol. 101, no. 1, pp. 15-25, 1997.

[4] H. Malchow, "Motional instabilities in prey-predator systems," Journal of Theoretical Biology, vol. 204, no. 4, pp. 639-647, 2000.

[5] D. V. Vayenas, G. Aggelis, V. Tsagou, and S. Pavlou, "Dynamics of a two-prey-one-predator system with predator switching regulated by a catabolic repression control-like mode," Ecological Modelling, vol. 186, no. 3, pp. 345-357, 2005.

[6] A. Verdy, "Modulation of predator-prey interactions by the Allee effect," Ecological Modelling, vol. 221, no. 8, pp. 1098-1107, 2010.

[7] M. M. Molina, M. A. Moreno-Armendáriz, and J. C. Seck Tuoh Mora, "On the spatial dynamics and oscillatory behavior of a predator-prey model based on cellular automata and local particle swarm optimization," Journal of Theoretical Biology, vol. 336, pp. 173-184, 2013.

[8] V. Křivan, "Behavioral refuges and predator-prey coexistence," Journal of Theoretical Biology, vol. 339, pp. 112-121, 2013.

[9] P. Turchin, Complex Population Dynamics: A Theoretical/ Empirical Synthesis, Princeton University Press, Princeton, NJ, USA, 2003.
[10] R. Xu, L. Chen, and F. Hao, "Periodic solutions of a discrete time Lotka-Volterra type food-chain model with delays," Applied Mathematics and Computation, vol. 171, no. 1, pp. 91-103, 2005.

[11] R. Xu and Z. Ma, "Stability and Hopf bifurcation in a ratiodependent predator-prey system with stage structure," Chaos, Solitons \& Fractals, vol. 38, no. 3, pp. 669-684, 2008.

[12] Z. Hu, G. Gao, and W. Ma, "Dynamics of a three-species ratiodependent diffusive model," Nonlinear Analysis: Real World Applications, vol. 11, no. 3, pp. 2106-2114, 2010.

[13] W. Li and L. Wang, "Stability and bifurcation of a delayed threelevel food chain model with Beddington-DeAngelis functional response," Nonlinear Analysis: Real World Applications, vol. 10, no. 4, pp. 2471-2477, 2009.

[14] Q. Gan, R. Xu, and P. Yang, "Bifurcation and chaos in a ratio-dependent predatory-prey system with time delay," Chaos, Solitons and Fractals, vol. 39, no. 4, pp. 1883-1895, 2009.

[15] T. C. Gard, Introduction to stochastic differential equations, Marcel Dekker, New York, NY, USA, 1988.

[16] R. M. May, Stability and Complexity in Model Ecosystems, Princeton University Press, Princeton, NJ, USA, 2001.

[17] M. P. Hassell and G. C. Varley, "New inductive population model for insect parasites and its bearing on biological control," Nature, vol. 223, no. 5211, pp. 1133-1137, 1969.

[18] J. R. Beddington, "Mutual interference between parasites or predators and its effect on searching efficiency," Journal of Animal Ecology, vol. 44, pp. 331-341, 1975.

[19] D. L. DeAngelis, R. A. Goldsten, and R. Neill, "A model for trophic interaction," Ecology, vol. 56, pp. 881-892, 1975.

[20] P. H. Crowley and E. K. Martin, "Functional response and interference within and between year classes of a dragonfly population," Journal of the North American Benthological Society, vol. 8, pp. 211-221, 1989.

[21] G. T. Skalski and J. F. Gilliam, "Functional responses with predator interference: viable alternatives to the Holling type II model," Ecology, vol. 82, no. 11, pp. 3083-3092, 2001.

[22] R. Z. Khasminskii, Stochastic Stability of Differential Equations, Sijthoff \& Noordhoff, Germantown, Md, USA, 1980.

[23] L. Arnold, Stochastic Differential Equations: Theory and Applications, John Wiley \& Sons, New York, NY, USA, 1972.

[24] A. Friedman, Stochastic Differential Equations and Their Applications, Academic Press, New York, NY, USA, 1976.

[25] X. Mao and C. Yuan, Stochastic Differential Equations with Markovian Switching, Imperial College Press, London, UK, 2006.

[26] X. Mao, Stochastic Differential Equations and Applications, Horwood Publishing, Chichester, UK, 2nd edition, 2007.

[27] M. B. Nevelson and R. Z. Khasminskii, Stochastic Approximation and Recurrent Estimation, Nauka, Moscow, Russia, 1972.

[28] H. Qiu, M. Liu, K. Wang, and Y. Wang, "Dynamics of a stochastic predator-prey system with Beddington-DeAngelis functional response," Applied Mathematics and Computation, vol. 219, no. 4, pp. 2303-2312, 2012.

[29] I. Karatzas and S. E. Shreve, Brownian Motion and Stochastic Calculus, Springer, Berlin, Germany, 1991.

[30] X. Mao, Stochastic Differential Equations and Applications, Horwood Publishing, Chichester, UK, 2008.

[31] I. Barbălat, "Systèmes d'équations différentielles d’oscillations non linéaires," Revue Roumaine de Mathematiques Pures et Appliquees, vol. 4, pp. 267-270, 1959. 
[32] X. Li and X. Mao, "Population dynamical behavior of nonautonomous Lotka-Volterra competitive system with random perturbation," Discrete and Continuous Dynamical Systems A, vol. 24, no. 2, pp. 523-545, 2009.

[33] D. J. Higham, "An algorithmic introduction to numerical simulation of stochastic differential equations," SIAM Review, vol. 43, no. 3, pp. 525-546, 2001. 


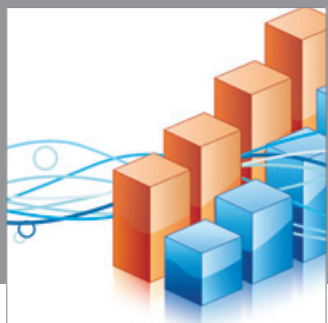

Advances in

Operations Research

mansans

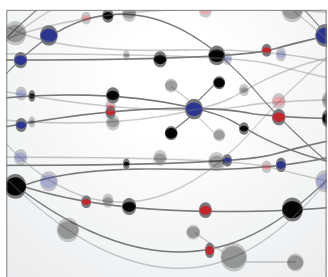

The Scientific World Journal
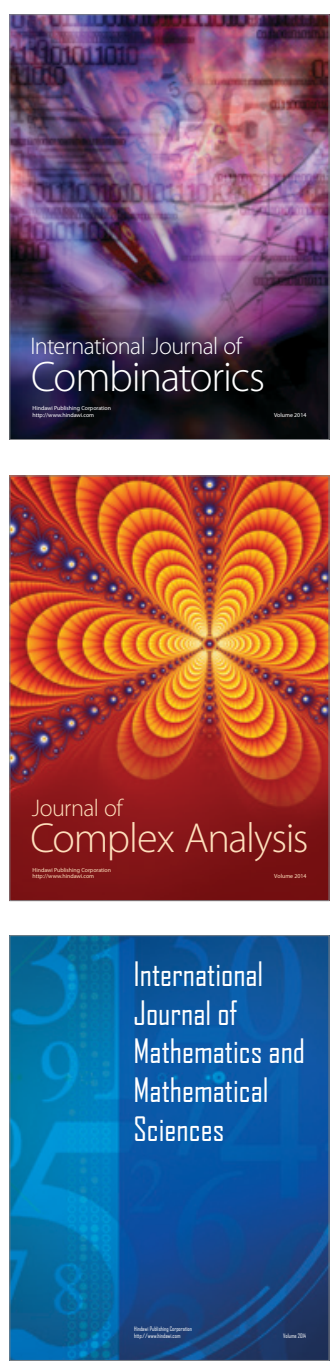
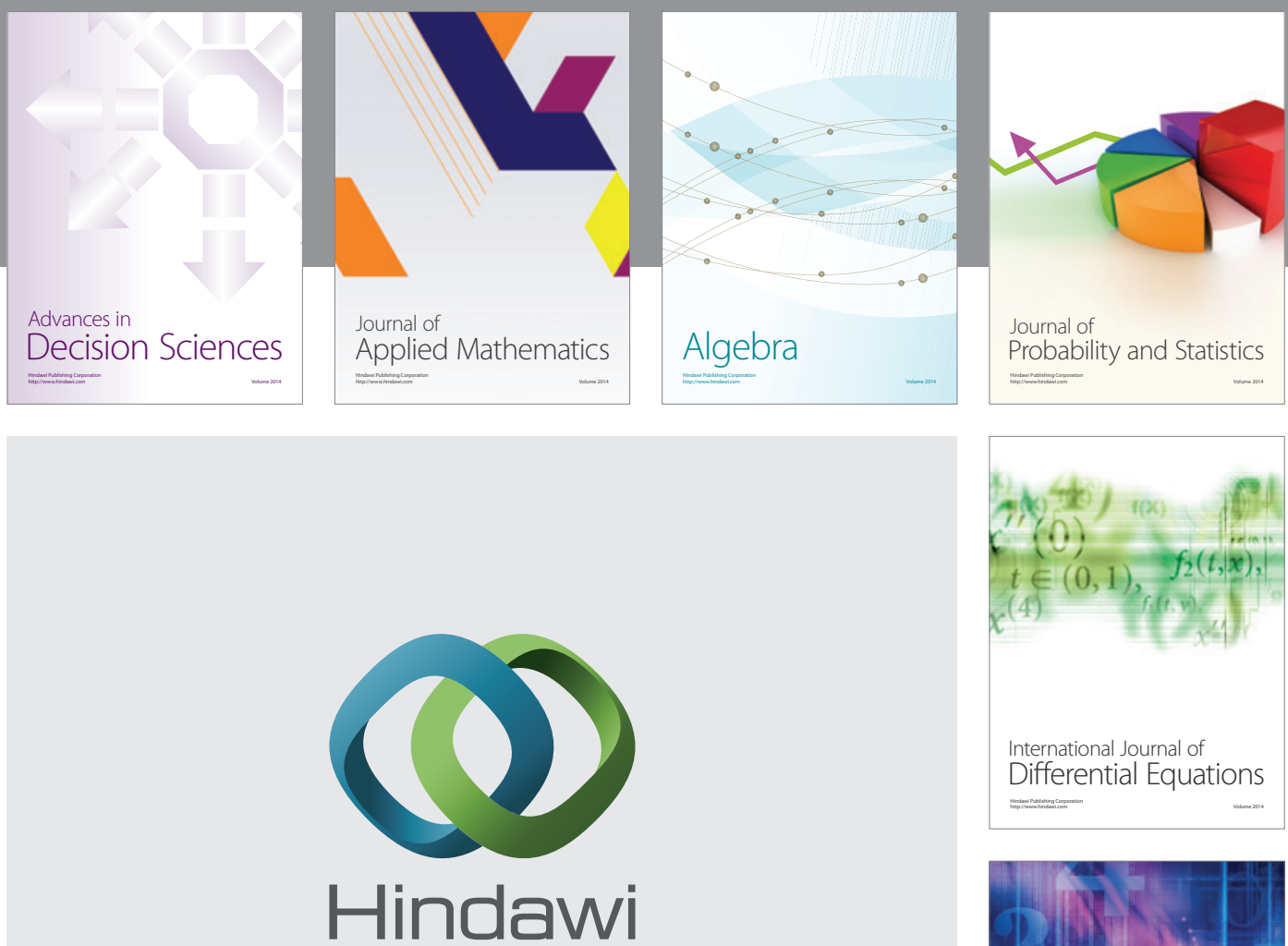

Submit your manuscripts at http://www.hindawi.com
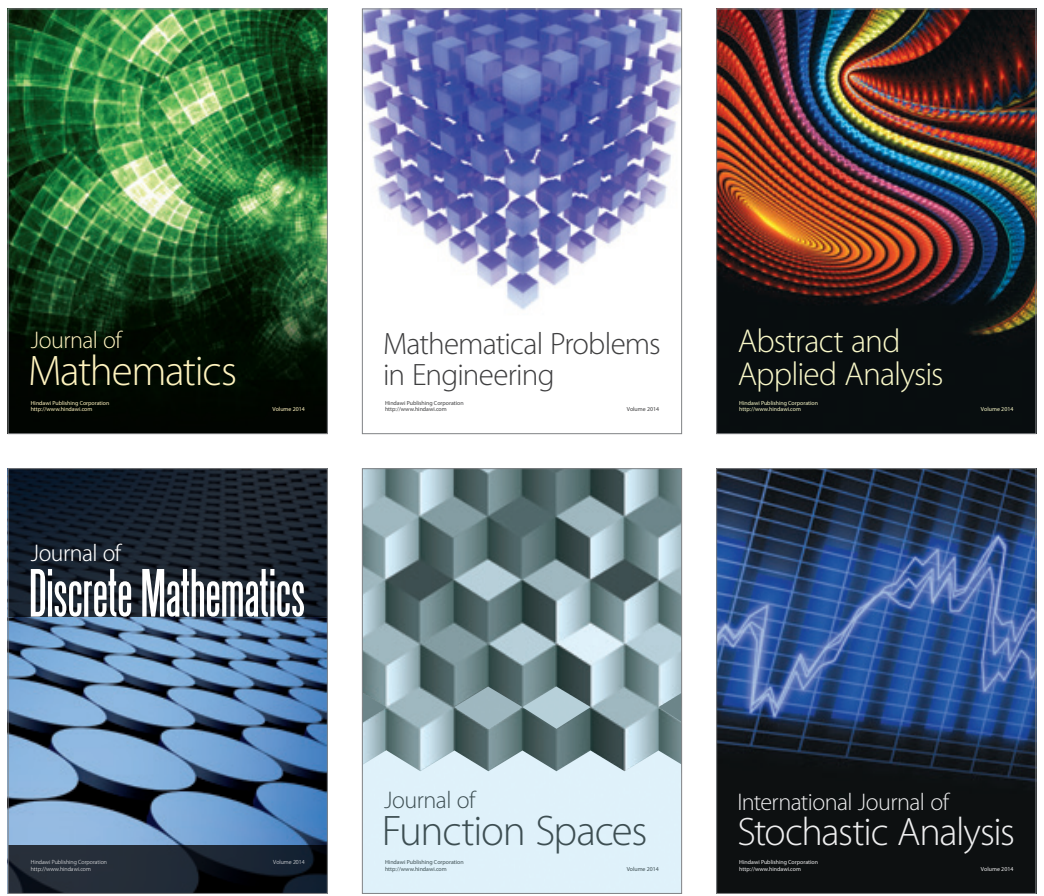

Journal of

Function Spaces

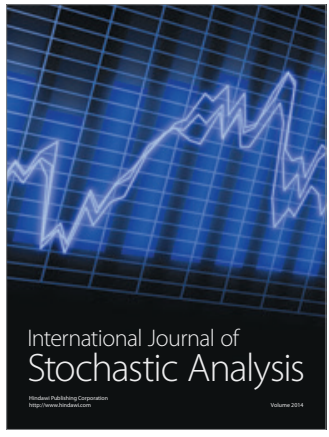

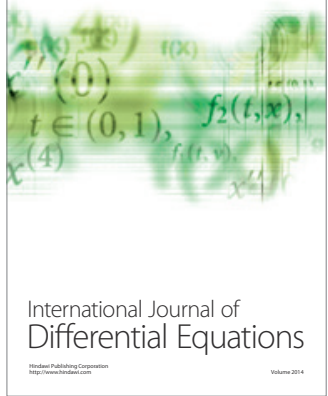
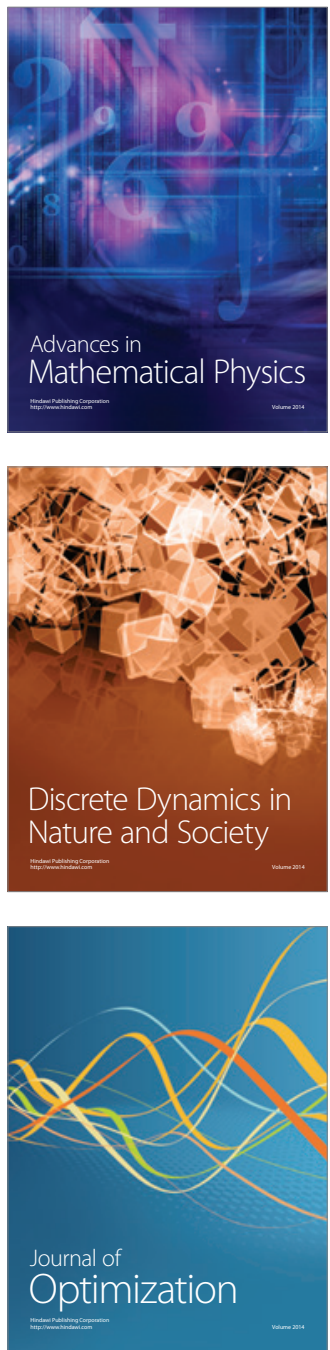